\title{
Ginsenoside panaxatriol reverses TNBC paclitaxel resistance by inhibiting the IRAK1/NF-KB and ERK pathways
}

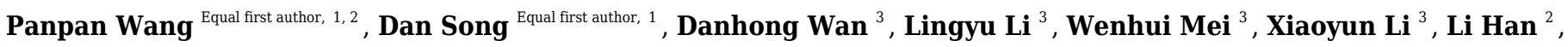 \\ Xiaofeng Zhu ${ }^{2}$, Li Yang ${ }^{1}$, Yu Cai ${ }^{\text {Corresp., } 1}$, Ronghua Zhang ${ }^{\text {Corresp. } 1}$ \\ ${ }^{1}$ College of Pharmacy, Jinan university, Guangzhou, China \\ 2 First Affiliated Hospital of Jinan University, Guangzhou, China \\ 3 College of Traditional Chinese Medicine, Jinan university, Guangzhou, China \\ Corresponding Authors: Yu Cai, Ronghua Zhang \\ Email address: tcaiyu@jnu.edu.cn, tzrh@jnu.edu.cn
}

Background. Paclitaxel (PTX) resistance is a major obstacle in the treatment of triplenegative breast cancer (TNBC). Previously, we have reported that interleukin-1 receptorassociated kinase 1 (IRAK1) and its downstream pathways are associated with PTX resistance in TNBC cells. In this study, we sought to investigate the combination treatment of ginsenoside panaxatriol (GPT), one of the main active components in Panax ginseng, with PTX on viability and apoptosis of TNBC PTX resistant cells, and explore the role of IRAK1 mediated signaling pathways in the therapeutic effects. Methods. CellTiter-Glo and colony formation assays were used to assess cell viability. Flow cytometry was used to analyze subG1 and apoptosis. Western blot was used to detect expressions of proteins involved in apoptosis and the IRAK1/NF-KB and ERK pathways. The mRNA expression of inflammatory cytokines, S100A7/8/9, and cancer stem cell (CSC) -related genes were examined by $\mathrm{qPCR}$. Stem cells were identified by tumor sphere assay. Cell invasion ability was examined by transwell assay. Results. We show that GPT inhibits MDA-MB-231 PTX resistant (MB231-PR) cell viability in a dose dependent manner. When combined with PTX, GPT synergistically causes more cell death, induces subG1 accumulation and cell apoptosis. Besides, up-regulation of $\mathrm{BAX} / \mathrm{BCL}-2$ ratio, and down-regulation of $\mathrm{MCL}-1$ are also observed. Moreover, this combination inhibit IRAK1, NF-KB and ERK1/2 activation, and lead to down-regulation of inflammatory cytokines (IL6, IL8, CXCL1, CCL2), S100A7/9, and CSC-related genes (OCT4, SOX2, NANOG, ALDH1, CD44) expression. In addition, the combination treatment suppresses MB231-PR cell invasion ability, and impairs tumor sphere growth both in MB231-PR and SUM159 PTX resistant (SUM159-PR) cells.

Conclusion. Our study demonstrates that GPT can resensitize TNBC PTX resistant cells to PTX by inhibiting the IRAK1/NF-KB and ERK pathways and reducing stem cell characteristics. 
1 Ginsenoside panaxatriol reverses TNBC paclitaxel

2 resistance by inhibiting the IRAK1/NF-KB and ERK

3 pathways

4 Panpan Wang ${ }^{1,2 \#}$, Dan Song ${ }^{1 \#}$, Danhong Wan ${ }^{3}$, Lingyu $\mathrm{Li}^{3}$,Wenhui Mei ${ }^{3}$, Xiaoyun Li ${ }^{3}, \mathrm{Li}^{3}$

5 Han $^{2}$, Xiaofeng Zhu ${ }^{2}$, Li Yang ${ }^{1}$, Yu Cai ${ }^{1 *}$, Ronghua Zhang ${ }^{1 *}$

6 1. College of Pharmacy, Jinan University, Guangzhou, Guangdong, 510630, PR China

7 2. First Affiliated Hospital of Jinan University, Guangzhou, Guangdong, 510630, PR China

8 3. College of Traditional Chinese Medicine, Jinan University, Guangzhou, Guangdong, 510630,

9 PR China

$10 *$ Correspondence:

11 Ronghua Zhang ${ }^{1}$; Yu Cai ${ }^{2}$

12 No.601, West Huangpu Avenue, Tianhe District, Guangzhou, Guangdong, 510630, PR China.

13 Email address: 1.tzrh@jnu.edu.cn; ${ }^{2 .}$ tcaiyu@jnu.edu.cn

14 \#: Co-first author

\section{Abstract}

Background. Paclitaxel (PTX) resistance is a major obstacle in the treatment of triplenegative breast cancer (TNBC). Previously, we have reported that interleukin-1 receptorassociated kinase 1 (IRAK1) and its downstream pathways are associated with PTX resistance in TNBC cells. In this study, we sought to investigate the combination treatment of ginsenoside panaxatriol (GPT), one of the main active components in Panax ginseng, with PTX on viability and apoptosis of TNBC PTX resistant cells, and explore the role of IRAK1 mediated signaling pathways in the therapeutic effects.

Methods. CellTiter-Glo and colony formation assays were used to assess cell viability. Flow cytometry was used to analyze subG1 and apoptosis. Western blot was used to detect expressions of proteins involved in apoptosis and the IRAK1/NF- $\kappa \mathrm{B}$ and ERK pathways. The mRNA expression of inflammatory cytokines, S100A7/8/9, and cancer stem cell (CSC) -related 
27 genes were examined by qPCR. Stem cells were identified by tumor sphere assay. Cell invasion 28 ability was examined by transwell assay.

29

30

31

32

Results. We show that GPT inhibits MDA-MB-231 PTX resistant (MB231-PR) cell viability in a dose dependent manner. When combined with PTX, GPT synergistically causes more cell death, induces subG1 accumulation and cell apoptosis. Besides, up-regulation of BAX/BCL-2 ratio, and down-regulation of MCL-1 are also observed. Moreover, this combination inhibit IRAK1, NF- $\kappa$ B and ERK1/2 activation, and lead to down-regulation of inflammatory cytokines (IL6, IL8, CXCL1, CCL2), S100A7/9, and CSC-related genes (OCT4, SOX2, NANOG, ALDH1, CD44) expression. In addition, the combination treatment suppresses MB231-PR cell invasion ability, and impairs tumor sphere growth both in MB231-PR and SUM159 PTX resistant (SUM159-PR) cells.

Conclusion. Our study demonstrates that GPT can resensitize TNBC PTX resistant cells to PTX by inhibiting the IRAK1/NF- $\kappa$ B and ERK pathways and reducing stem cell characteristics.

\section{Introduction}

TNBC is a highly invasive subtype of breast cancer with poor prognosis (Foulkes et al. 2010). Because of the lack of hormone receptors and human epidermal growth factor receptor 2 (HER2) amplification, TNBC does not respond to hormone or anti-HER2 treatment, and mainly relies on traditional chemotherapy (Denkert et al. 2017). PTX-based chemotherapy regimens are the most widely used first-line therapeutic strategies for clinically treatment of TNBC. Although effective in the initial treatment, a subset of patients eventually develops resistance, and leads to disease progression (Mustacchi \& De Laurentiis 2015; Schettini et al. 2016). Hence, it is highly necessary to find a solution for PTX resistance in TNBC.

The nuclear factor kappa $\mathrm{B}(\mathrm{NF}-\kappa \mathrm{B})$ signaling pathway plays an important role in cancer initiation, progression, and resistance, thus making it a good target for cancer treatment (Chaturvedi et al. 2011; Hoesel \& Schmid 2013; Taniguchi \& Karin 2018). However, despite numerous attempts to develop molecular drugs that specifically target NF- $\kappa \mathrm{B}$, few clinical advancements have been made (Baud \& Karin 2009). Previously, by using gain and loss of function methods, we reported that activation of interleukin-1 receptor-associated kinase 1 (IRAK1), an upstream kinase of the NF- $\mathrm{B}$ signaling pathway, is associated with PTX resistance 
56 in TNBC cells (Wee et al. 2015). Importantly, together with S100A7, S100A8 and S100A9

57 (S100A7/8/9), IRAK1 form a druggable circuitry which drives the malignancy of TNBC cells

58 (Goh et al. 2017). These observations prompted us to search for potential candidate drugs that

59 can target IRAK1 and its downstream signaling pathways.

60 Ginseng and its active ingredient ginsenosides, such as ginsenosides Rg3 (GRg3), have been

widely used in China to treat cancers in the clinic. Ginsenosides are a class of steroid glycosides

62 and triterpene saponins. Over the last decade, more than 100 different types have been isolated and identified. Researchers have found that GRg3 can facilitate the penetration of PTX through the Caco-2 monolayer from the apical side to the basal side, and enhance the oral bioavailability of PTX in vivo (Yang et al. 2012). Furthermore, GRg3 can inhibit P-glycoprotein expression and increase the accumulation of drugs such as vincristine in multidrug resistant cells, but not in sensitive cells (Kim et al. 2003). Importantly, it has been reported that some ginsenosides can inhibit the activation of IRAK1 and its downstream pathways (Joh et al. 2011; Nag et al. 2012; Shaukat et al. 2019). In this study, we investigated the in vitro anti-viability of GPT in TNBC PTX resistant cells, and found that GPT can target IRAK1/NF- $\mathrm{kB}$ and ERK pathways to overcome resistance.

\section{Materials \& Methods}

\section{Chemicals and reagents}

GPT was obtained from Must Bio-Technology (Chengdu, China). PTX was purchased from Sigma-Aldrich (St. Louis, MO, USA). Dulbecco's Modified Eagle Medium (DMEM) (11995040), F-12 nutrient mixture (Ham) and fetal bovine serum (FBS) were bought from Life Technologies (Grand Island, NY, USA). MammoCul medium (human) and supplements were purchased from STEMCELL Technologies (Vancouver, Canada). CellTiter-Glo luminescent cell viability assay kits were purchased from Promega Corporation (Madison, WI, USA). iScript gDNA Clear cDNA Synthesis Kits and iTaq Universal SYBR Green Supermix Kits were purchased from Bio-Rad Laboratories (Hercules, CA USA). p-IRAK1 S376, IRAK1, p-P65 S536, P65, p-ERK1/2, ERK1/2, BAX, BCL-2 and MCL-1 antibodies were supplied by Cell Signaling Technology (Danvers, MA, USA). Beta-actin antibody was purchased from Sigma Aldrich (St. Louis, MO, USA). 


\section{Cell culture and viability assay}

MB231 cells and SUM159 cells were obtained from ATCC. MB231-PR cells and SUM159-PR were established as previously described (Wee et al. 2015). Briefly, cells were treated with increasing concentrations of PTX for over a period of 3 months. Then, MB231-PR cells were cultured in DMEM supplemented with 75 nM PTX, penicillin/streptomycin, and 10\% FBS at 37 ${ }^{\circ} \mathrm{C}$ with $5 \% \mathrm{CO}_{2}$. SUM159-PR cells were maintained in F-12 supplemented with 300nM paclitaxel, 5\% FBS, $10 \mathrm{mM}$ HEPES, $10 \mu \mathrm{g} / \mathrm{ml}$ hydrocortisone, $5 \mu \mathrm{g} / \mathrm{ml}$ insulin and 1\% penicillin/streptomycin. For cell viability assay, 1000 cells/well in $90 \mu \mathrm{l}$ medium were seeded into Costar 96-well white plates. The next day, different concentrations of drugs in $10 \mu \mathrm{l}$ medium were added and incubated for the indicated times. Then, cells were lysed with $50 \mu$ CellTiterGlo reagent and the chemiluminescent signals were detected with a PerkinElmer VICTOR X4 plate reader.

\section{Cell cycle and apoptosis assay}

Cell cycle and apoptosis analysis was performed by DNA content quantification to quantify the subG1 population, which is a reflective of the extent of cell death. Briefly, floating and adherent cells were harvested together after $48 \mathrm{~h}$ and $72 \mathrm{~h}$ treatment respectively. Then, cells were fixed by $70 \%$ ethanol at $4{ }^{\circ} \mathrm{C}$ overnight. After washing with phosphate buffered saline (PBS), cells were resuspended in $100 \mu \mathrm{l}$ of $100 \mu \mathrm{g} / \mathrm{ml}$ RNase A. 5 min later, $400 \mu \mathrm{l}$ of $50 \mu \mathrm{g} / \mathrm{ml}$ propidium iodide were added, and cells were incubated for $30 \mathrm{~min}$ in dark area. Finally, the stained cells were analyzed by FACScalibur and quantified using CellQuest software.

\section{Colony formation assay}

1000 cells/well were seeded into 12 -well plates. The next day, drugs were added and incubated for 12 days. Medium was changed every 3 days. Then, cells were washed with PBS and fixed with methanol for $10 \mathrm{~min}$. Finally, cells were then stained with $0.1 \%$ crystal violet at room temperature for $10 \mathrm{~min}$ and photographed. 


\section{Tumor sphere formation assay}

1113000 cells/well in $180 \mu \mathrm{l}$ medium were seeded into Corning 96-well spheroid microplates in 112 complete MammoCul medium. The next day, drugs in $20 \mu 1$ medium were added and incubated 113 for 12 days. Pictures were taken on day 6 and day 12. Finally, on day 12, cells were lysed with $114100 \mu \mathrm{l}$ CellTiter-Glo reagent and the chemiluminescent signal was detected with a PerkinElmer 115 VICTOR X4 plate reader.

\section{Transwell invasion assay}

11710000 cells in $100 \mu \mathrm{l}$ serum-free DMEM containing DMSO, PTX, GPT, or combination were 118 added into Corning Transwell polycarbonate membrane inserts coated with Matrigel (300 $119 \mu \mathrm{g} / \mathrm{mL}$ ). And medium containing 10\% FBS was added to the bottom chamber. After $24 \mathrm{~h}$ 120 incubation, the cells that remained on the above surface of the insert membrane were scraped off 121 with a cotton swab. The cells that passed through Matrigel to the bottom of the insert were fixed 122 with paraformaldehyde and stained with $0.1 \%$ crystal violet in methanol. The inserts were 123 photographed, and the cells were counted.

\section{Quantitative-PCR (qPCR) assay}

125 RNA extraction and purification were performed according to the instructions from Zymo 126 Research (R2052). 750 ng RNA was used to synthesize cDNA. And qPCR was performed using 127 the Applied Biosystems 7500 Fast Real-Time PCR system. All primers are listed in table 1. For 128 quantification of mRNA levels, 18S was used as the internal control, and the expression of target 129 genes were analyzed using the $2^{-\Delta \Delta C T}$ method.

\section{Western blot assay}

131 Western blot was performed using whole-cell extracts in protein lysis buffer with freshly added 132 protease inhibitor cocktail. Proteins were separated on 8\%-10\% SDS polyacrylamide gel 133 electrophoresis gels and transferred to polyvinylidene difluoride membranes. The membrane was 134 blocked with 5\% non-fat dry milk in tris-buffered saline (TBS) containing $0.1 \%$ Tween 20 135 (TBST). The membrane was then incubated with primary antibody (1:1000 dilution) in 5\% 136 bovine serum albumin overnight. After washed 3 times with TBST, the membrane was incubated 
137 with secondary antibody (1:2000 dilution) in 5\% non-fat dry milk at room temperature for $1 \mathrm{~h}$. 138 Then, SuperSignal West Femto Maximum Sensitivity Substrate was added, and images were 139 taken using the ChemiDoc MP System.

\section{Statistical analysis}

141 Data are shown as mean $\pm \mathrm{SD}$. The $\mathrm{t}$ test was used to determine whether there are any

142 statistically significant differences between two groups. $P<0.05$ was considered statistically 143 significant.

\section{Results}

\section{GPT promotes cytotoxicity of PTX in MB231-PR cells}

146 To explore whether GPT can promote cytotoxicity of PTX in TNBC resistant cells, MB231-PR

147 was constructed and used as cell model. Firstly, we conducted CellTiter-Glo assay to observe

148 different concentration of GPT on cell viability. As shown in Fig. 1A, GPT treatment

149 significantly decreased cell viability of MB231-PR cells in a dose dependent manner, with the

150 half maximal inhibitory concentration (IC50) $21.39 \mu \mathrm{M}$. Secondly, we combined GPT with PTX

151 to check whether they have synergistic effects. Results showed that the combination caused

152 dramatic cell death in a dose and time dependent manner, comparing to either single use group

153 (Fig. 1B). Interestingly, the synergistic effects didn’t apply to MB231 parental (MB231-PT)

154 cells, although MB231-PT cells were sensitive to PTX (Fig. S1) and showed more sensitive to

155 GPT when treated with the same concentration (Fig. 1C). Notable, the clinical using drug GRg3

156 didn't cause significant cell death in single or combination treatment group (Fig. S2). In addition,

157 colony formation assay confirmed the synergistic cytotoxicity effects of the combination on

158 MB231-PR cells (Fig. 1D, Fig. S3).

159 Since chemotherapy resistance appears partly due to aberrant changes of signaling pathways that

160 endowed cells with the abilities to escape apoptosis, restoring apoptosis is a very important 161 therapeutic strategy for antitumor therapy (Baig et al. 2016; Plati et al. 2008). Therefore, next, 162 we used flow cytometry to measure subG1 changes after the combination treatment, which is 163 marker of apoptosis. Not surprisingly, GPT combined with PTX significantly increased subG1 
164 cell accumulation both after $48 \mathrm{~h}$ and $72 \mathrm{~h}$ (Fig. 1E, Fig. S4). Taken together, these results

165 suggested GPT as a very effective molecular to reverse PTX resistance in TNBC cells.

\section{The combination treatment activates mitochondria mediated apoptosis}

167 The alteration of pro-apoptotic proteins and anti-apoptotic proteins play important roles in the 168 determination of cancer cells apoptosis, and are associated with chemoresistance (Campbell \& 169 Tait 2018; Warren et al. 2019). Thus, we observed the protein expression of BAX and BCL-2 170 after treatment, two key mediators of apoptotic response to chemotherapy. As shown in Fig 2A171 B, GPT combined with PTX significantly increased BAX and decreased BCL-2 expression in a 172 dose and time dependent manner.

173 Besides BAX and BCL-2, MCL-1 was recently reported to be associated with poor prognosis in 174 TNBC patients and can be used as a therapeutic target (Campbell et al. 2018). Notably, we have 175 shown that IRAK1 inhibitor can decrease MCL-1 expression in MB321-PR cells to induce cell 176 apoptosis (Wee et al. 2015). Therefore, we also evaluated the protein expression of MCL-1 after 177 treatment. As shown in Fig. 2A-B, the combination treatment also resulted in down-regulation of 178 MCL-1 expression. These results together suggested that the combination treatment activated 179 mitochondria mediated apoptosis to reverse PTX resistance.

180

181

182

183

184

185

186

187

188

189

190

191

\section{The combination treatment inhibits IRAK1/NF-KB and ERK pathways}

To further clarify the signaling pathways that involved in GPT effects, gene expression profiling was conducted in MB231-PR cells treated with DMSO, PTX, GPT, and combination, respectively. Results showed that NOD-like receptor signaling pathways played an important part in GPT activity in MB231-PR cells (data not shown). Interestingly, through loss and gain of function study, we have previously reported that activation of IRAK1, a key kinase of NOD-like receptor signaling pathway, is associated with PTX resistance in TNBC cells (Wee et al. 2015) Moreover, target IRAK1 using pharmacologic inhibitor can induce MB231-PR cells apoptosis, when combined with PTX (Wee et al. 2015) Thus, consideration was given to IRAK1 and its downstream signaling pathways. Results showed that the combination treatment can significantly inhibit the phosphorylation of IRAK1, P65, ERK1/2, and increase the expression of IкB-alpha in a dose and time dependent manner (Fig. 2A-B). 
192 To additionally characterize the functional effects of IRAK1 mediated pathways, we investigated 193 the mRNA expression of NF-кB target genes by qPCR, including interleukin 6 (IL6), IL8, 194 chemokine (C-X-C motif) ligand 1 (CXCL1), and chemokine (C-C motif) ligand 2 (CCL2). The 195 above cytokines were shown to be distinctly expressed among different group in our gene 196 expression profiling experiment, and were reported to be critical for the anchorage independent 197 growth of TNBC cells (Hartman et al. 2013). As shown in Fig. 2C-F, (Table S1, Table S3), 198 compared to DMSO, PTX significantly promoted the expression of IL6, IL8, CXCL1 and CCL2. 199 However, this induction can be significantly attenuated when combined with GPT.

200 Except these target cytokines, we previously published that IRAK1 and S100A7/8/9 form a 201 feedback loop to drive the malignancy of TNBC cells (Goh et al. 2017). Here, we also showed 202 that the combination treatment significantly decreased S100A7 and S100A9 mRNA expression 203 (Fig. 2G-H, Table S1, Table S3), although S100A8 mRNA expression level was too low to 204 detect. These results together suggested that the combination treatment overcome PTX resistance 205 by inhibiting IRAK1 mediated NF- $\mathrm{B}$ and ERK pathways.

206 The combination treatment inhibits CSC-related genes expression and impairs tumor 207 sphere growth and invasion ability

208 Accompanied with killing cancer cell, PTX treatment has been reported to induce CSC 209 enrichment, another key mechanism suggested to be responsible for chemoresistance and cancer 210 metastasis (Bousquet et al. 2017; Zhang et al. 2019). And drug that can target cancer stemness

211 are proposed as new strategies for clinical cancer treatment (Saygin et al. 2019; Sun et al. 2019).

212 In order to testify the effect of combination therapy on characteristics of CSC, firstly, qPCR was 213 used to check the expression of a group of CSC-related genes. As shown in Fig. 3A-E, (Table 214 S2, Table S4, Table S5), compared to PTX, the combination treatment significantly lead to 215 down-regulation of octamer-binding transcription factor 4 (OCT4), sex determining region Y216 box 2 (SOX2), NANOG, aldehyde dehydrogenase 1 (ALDH1), and CD44 gene expression.

217 Secondly, transwell invasion and tumor sphere assay were conducted to assess CSC properties. 218 As shown in Fig. 3F and 3G-J, the combination treatment significantly suppressed MB231-PR 219 cell invasion ability, and impaired tumor sphere growth both in MB231-PR and SUM159-PR 220 cells. 


\section{Discussion}

222 Treatment of TNBC has been challenging, due to lack of target therapy options and constantly

223 acquired resistance. Therefore, a combinatorial therapy has been always preferred to achieve a 224 synergistic effects. Plant derived compounds, such as saponins, flavonoids and alkaloids, have

225 been tested and proved to be effective in killing cancer cells and restoring resistant cells to 226 chemotherapy (Aung et al. 2017). Of which, ginsenosides have been researched in different 227 cancers. Results showed that GRg3 can enhance the anti-cancer ability of chemo drugs by modulating the oral bioavailability (Yang et al. 2012), inhibiting P-glycoprotein expression (Kim et al. 2003), inhibiting cell autophagy (Wang et al. 2019), and down regulating epidermal growth factor receptor (EGFR)/ phosphatidylinositol-3-kinase (PI3K)/ Akt signaling pathway (Jiang et al. 2017). In this study, we investigated the combination treatment of GPT and PTX on viability and apoptosis of TNBC PTX resistant cells, and clarified the signaling pathways underlies. Our data showed that the combination can synergistically inhibit MB231-PR cell viability, induce subG1 accumulation and trigger the mitochondrial mediated apoptosis. Our data further suggested that the combination can inhibit IRAK1/NF- $\mathrm{kB}$ and ERK signaling pathways, resulted in down-regulation of inflammatory factors and S100A7/9 expression, which are the main cytokines in tumor microenvironment contributed to CSC phenotype and function. In addition, we showed that combination can inhibit CSC-related genes expression and impair invasion ability and tumor sphere growth.

240 It is suggested that the BCL-2 family are key mediators of anti-cancer therapeutics, and abnormal expression of apoptotic proteins contributed to chemoresistance (Hata et al. 2015). In addition to other members, decreased BAX/BCL-2 ratio and elevated MCL-1 expression were reported to be closely related with PTX resistance in breast cancer (Lee et al. 2017; Sharifi et al. 2014). Drugs which can inhibit the activity of these proteins are believed to improve the efficacy of chemotherapeutic agents. Interestingly, our data showed that GPT augments the effects of PTX by up-regulating BAX/BCL-2 ratio and down-regulating MCL-1 expression.

247 The results in this study are consistent with our previous published papers, showing that 248 pharmacologic inhibition of IRAK1 phosphorylation and downstream signaling pathways activation can overcome TNBC PTX resistance. Notably, other group recently reported that the expression of IRAK1 was positively correlated with tumor size following neoadjuvant chemotherapy (NCT) (Yang et al. 2019). Breast cancer patients, with higher expression of 
252 IRAK1 both before and after NCT, had a shorter survival period (Yang et al. 2019). These 253 results together highlight the role of IRAK1 in chemoresistance and clinical application of

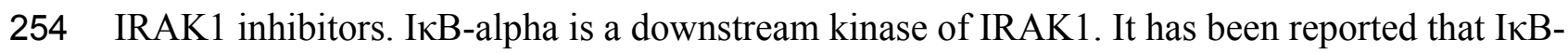
255 alpha plays an important role in NF- $\kappa$ B cytosolic-nuclear translocation. I $\kappa$ B-alpha enters the 256 nucleus to bind NF- $\mathrm{BB}$ dimers and translocate them to the cytosol(Christian et al. 2016). 257 Researchers also showed that I $\kappa$ B-alpha was the key mediator responsible for PTX induced NF$258 \kappa \mathrm{B}$ nuclear translocation, DNA binding and transcriptional activity (Huang et al. 2000). 259 Consistently, decreased I $\kappa$ B-alpha and increased NF- $\kappa$ B transcriptional activity after PTX 260 treatment can also be seen in our experiment. However, the combination treatment increased 261 I $\kappa$ B-alpha expression and decreased NF- $\kappa B$ transcriptional activity.

262 Another our major finding is that inhibition of IRAK1/NF- $\kappa$ B and ERK pathways by GPT 263 reduced stem cell characteristics. CSCs have been reported as one of the determining reasons for 264 chemoresistance and subsequent cancer relapse. And one of the mechanisms that CSCs are 265 acquired is taking advantage of PTX treatment induced inflammation cytokines and S100 protein 266 family in tumor microenvironment.

267 In our experiment, decreased expression of inflammation cytokines (IL-6, IL-8, CXCL1 and 268 CCL2) can be noticed in the combination group. The above cytokines are reported to be NF- $\kappa \mathrm{B}$ 269 transcriptional targets, and their expression are induced following NF- $\kappa$ B activation after chemo 270 treatment (Jia et al. 2017). In turn, these factors activate inflammation related signaling pathways 271 such as NF-kB and signal transducer and activator of transcription 3 (STAT3) (Wang et al. 2018; 272 Wong et al. 2015; Yue et al. 2006), which further promote cell survival through regulating 273 apoptosis proteins and promote the formation of CSC through regulating CSC related genes 274 (Rhyasen et al. 2013). Importantly, in accordance to IL-8 inhibitor, anti-IL6 antibody, anti275 CXCL1 antibody, or anti-CCL2 antibody, here we showed that target IRAK1 mediated pathways 276 by ginsenoside PPT can effectively down-regulate these cytokines and disrupt this process (Dey 277 et al. 2019; Heo et al. 2016; Miyake et al. 2019; Teng et al. 2017).

278 Besides, we also identified that S100A7/9 were down-regulated after combination treatment. $279 \mathrm{~S} 100 \mathrm{~A} 7 / 9$ are members of the S100 protein family, which are closely related to tumorigenesis 280 and progression (Cancemi et al. 2018; Chen et al. 2014). In addition, S100A7/8/9 can be

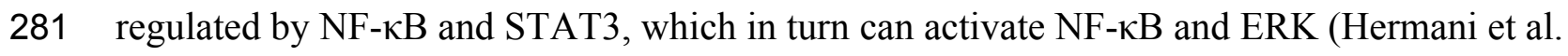
282 2006; Liu et al. 2013; Nemeth et al. 2009). S100A8/9 and CXCL1/2, or S100A7/8/9 and IRAK1, 
283 form a feedback loop to cause cancer chemoresistance and drive breast cancer tumor sphere 284 growth (Acharyya et al. 2012; Goh et al. 2017). Collectively, our data suggested that GPT can 285 disrupt this feedback loop to inhibit CSC characteristics.

286 As to molecular phenotype in breast cancer, CSCs display CD44+/CD24- phenotype and high

287 ALDH1 activity. In parallel, other characters include overexpression of transcription factors

288 OCT4, SOX2 and NANOG, which are associated with high-grade stage and poor clinical 289 outcome in TNBC. In this part, we demonstrated that GPT combined with PTX can inhibit CSCs 290 related gene expression, impair invasion ability and tumor sphere growth.

291

292

293

294

295

296

297

298

299

300

301

302

303

304

305

306

307

308

309

310

311

312

313

314

315

316

317

318

\section{Conclusions}

Our study demonstrates that GPT can resensitize TNBC PTX resistant cells to PTX treatment by inhibiting the IRAK1/NF- $\mathrm{BB}$ and ERK pathways, reducing stem cell characteristics, thus provide it as a novel molecular for clinic use.

\section{Acknowledgements}

We thank all the reviewers for their helpful comments.

\section{References}

Acharyya S, Oskarsson T, Vanharanta S, Malladi S, Kim J, Morris PG, Manova-Todorova K, Leversha M, Hogg N, Seshan VE, Norton L, Brogi E, and Massague J. 2012. A CXCL1 paracrine network links cancer chemoresistance and metastasis. Cell 150:165-178. 10.1016/j.cell.2012.04.042

Aung TN, Qu Z, Kortschak RD, and Adelson DL. 2017. Understanding the Effectiveness of Natural Compound Mixtures in Cancer through Their Molecular Mode of Action. Int J Mol Sci 18. 10.3390/ijms18030656

Baig S, Seevasant I, Mohamad J, Mukheem A, Huri HZ, and Kamarul T. 2016. Potential of apoptotic pathway-targeted cancer therapeutic research: Where do we stand? Cell Death Dis 7:e2058. 10.1038/cddis.2015.275

Baud V, and Karin M. 2009. Is NF-kappaB a good target for cancer therapy? Hopes and pitfalls. Nat Rev Drug Discov 8:33-40. 10.1038/nrd2781

Bousquet G, El Bouchtaoui M, Sophie T, Leboeuf C, de Bazelaire C, Ratajczak P, Giacchetti S, de Roquancourt A, Bertheau P, Verneuil L, Feugeas JP, Espie M, and Janin A. 2017. Targeting autophagic cancer stem-cells to reverse chemoresistance in human triple negative breast cancer. Oncotarget 8:35205-35221. 10.18632/oncotarget.16925

Campbell KJ, Dhayade S, Ferrari N, Sims AH, Johnson E, Mason SM, Dickson A, Ryan KM, Kalna G, Edwards J, Tait SWG, and Blyth K. 2018. MCL-1 is a prognostic indicator and drug target in breast cancer. Cell Death Dis 9:19. 10.1038/s41419-017-0035-2

Campbell KJ, and Tait SWG. 2018. Targeting BCL-2 regulated apoptosis in cancer. Open Biol 8. $10.1098 /$ rsob.180002 
319

320

321

322

323

324

325

326

327

328

329

330

331

332

333

334

335

336

337

338

339

340

341

342

343

344

345

346

347

348

349

350

351

352

353

354

355

356

357

358

359

360

361

362

363

364

365

366

367

Cancemi P, Buttacavoli M, Di Cara G, Albanese NN, Bivona S, Pucci-Minafra I, and Feo S. 2018. A multiomics analysis of $\mathrm{S} 100$ protein family in breast cancer. Oncotarget 9:29064-29081. 10.18632/oncotarget.25561

Chaturvedi MM, Sung B, Yadav VR, Kannappan R, and Aggarwal BB. 2011. NF-kappaB addiction and its role in cancer: 'one size does not fit all'. Oncogene 30:1615-1630. 10.1038/onc.2010.566

Chen H, Xu C, Jin Q, and Liu Z. 2014. S100 protein family in human cancer. Am J Cancer Res 4:89-115.

Christian F, Smith EL, and Carmody RJ. 2016. The Regulation of NF-kappaB Subunits by Phosphorylation. Cells 5. 10.3390/cells5010012

Denkert C, Liedtke C, Tutt A, and von Minckwitz G. 2017. Molecular alterations in triple-negative breast cancer-the road to new treatment strategies. Lancet 389:2430-2442. 10.1016/s0140-6736(16)32454-0

Dey P, Rathod M, and De A. 2019. Targeting stem cells in the realm of drug-resistant breast cancer. Breast Cancer (Dove Med Press) 11:115-135. 10.2147/bctt.S189224

Foulkes WD, Smith IE, and Reis-Filho JS. 2010. Triple-negative breast cancer. N Engl J Med 363:1938-1948. 10.1056/NEJMra1001389

Goh JY, Feng M, Wang W, Oguz G, Yatim S, Lee PL, Bao Y, Lim TH, Wang P, Tam WL, Kodahl AR, Lyng MB, Sarma S, Lin SY, Lezhava A, Yap YS, Lim AST, Hoon DSB, Ditzel HJ, Lee SC, Tan EY, and Yu Q. 2017. Chromosome 1q21.3 amplification is a trackable biomarker and actionable target for breast cancer recurrence. Nat Med 23:1319-1330. $10.1038 / \mathrm{nm} .4405$

Hartman ZC, Poage GM, den Hollander P, Tsimelzon A, Hill J, Panupinthu N, Zhang Y, Mazumdar A, Hilsenbeck SG, Mills GB, and Brown PH. 2013. Growth of triple-negative breast cancer cells relies upon coordinate autocrine expression of the proinflammatory cytokines IL-6 and IL-8. Cancer Res 73:3470-3480. 10.1158/0008-5472.Can-12-4524-t

Hata AN, Engelman JA, and Faber AC. 2015. The BCL2 Family: Key Mediators of the Apoptotic Response to Targeted Anticancer Therapeutics. Cancer Discov 5:475-487. 10.1158/2159-8290.Cd-15-0011

Heo TH, Wahler J, and Suh N. 2016. Potential therapeutic implications of IL-6/IL-6R/gp130targeting agents in breast cancer. Oncotarget 7:15460-15473. 10.18632/oncotarget.7102

Hermani A, De Servi B, Medunjanin S, Tessier PA, and Mayer D. 2006. S100A8 and S100A9 activate MAP kinase and NF-kappaB signaling pathways and trigger translocation of RAGE in human prostate cancer cells. Exp Cell Res 312:184-197. 10.1016/j.yexcr.2005.10.013

Hoesel B, and Schmid JA. 2013. The complexity of NF-kappaB signaling in inflammation and cancer. Mol Cancer 12:86. 10.1186/1476-4598-12-86

Huang Y, Johnson KR, Norris JS, and Fan W. 2000. Nuclear factor-kappaB/lkappaB signaling pathway may contribute to the mediation of paclitaxel-induced apoptosis in solid tumor cells. Cancer Res 60:4426-4432.

Jia D, Li L, Andrew S, Allan D, Li X, Lee J, Ji G, Yao Z, Gadde S, Figeys D, and Wang L. 2017. An autocrine inflammatory forward-feedback loop after chemotherapy withdrawal facilitates the repopulation of drug-resistant breast cancer cells. Cell Death Dis 8:e2932. 10.1038/cddis.2017.319

Jiang J, Yuan Z, Sun Y, Bu Y, Li W, and Fei Z. 2017. Ginsenoside Rg3 enhances the antiproliferative activity of erlotinib in pancreatic cancer cell lines by downregulation of EGFR/PI3K/Akt signaling pathway. Biomed Pharmacother 96:619-625. 10.1016/j.biopha.2017.10.043

PeerJ reviewing PDF | (2020:01:45012:2:0:NEW 3 May 2020) 
368

369

370

371

372

373

374

375

376

377

378

379

380

381

382

383

384

385

386

387

388

389

390

391

392

393

394

395

396

397

398

399

400

401

402

403

404

405

406

407

408

409

410

411

412

413

414

415

416

417

Joh $\mathrm{EH}$, Lee IA, Jung IH, and Kim DH. 2011. Ginsenoside Rb1 and its metabolite compound K inhibit IRAK-1 activation--the key step of inflammation. Biochem Pharmacol 82:278-286. 10.1016/j.bcp.2011.05.003

Kim SW, Kwon HY, Chi DW, Shim JH, Park JD, Lee YH, Pyo S, and Rhee DK. 2003. Reversal of P-glycoprotein-mediated multidrug resistance by ginsenoside $\mathrm{Rg}(3)$. Biochem Pharmacol 65:75-82. 10.1016/s0006-2952(02)01446-6

Lee KM, Giltnane JM, Balko JM, Schwarz LJ, Guerrero-Zotano AL, Hutchinson KE, Nixon MJ, Estrada MV, Sanchez V, Sanders ME, Lee T, Gomez H, Lluch A, Perez-Fidalgo JA, Wolf MM, Andrejeva G, Rathmell JC, Fesik SW, and Arteaga CL. 2017. MYC and MCL1 Cooperatively Promote Chemotherapy-Resistant Breast Cancer Stem Cells via Regulation of Mitochondrial Oxidative Phosphorylation. Cell Metab 26:633-647.e637. 10.1016/j.cmet.2017.09.009

Liu H, Wang L, Wang X, Cao Z, Yang Q, and Zhang K. 2013. S100A7 enhances invasion of human breast cancer MDA-MB-468 cells through activation of nuclear factor-kappaB signaling. World J Surg Oncol 11:93. 10.1186/1477-7819-11-93

Miyake M, Furuya H, Onishi S, Hokutan K, Anai S, Chan O, Shi S, Fujimoto K, Goodison S, Cai W, and Rosser CJ. 2019. Monoclonal Antibody against CXCL1 (HL2401) as a Novel Agent in Suppressing IL6 Expression and Tumoral Growth. Theranostics 9:853-867. $10.7150 /$ thno.29553

Mustacchi G, and De Laurentiis M. 2015. The role of taxanes in triple-negative breast cancer: literature review. Drug Des Devel Ther 9:4303-4318. 10.2147/dddt.S86105

Nag SA, Qin JJ, Wang W, Wang MH, Wang H, and Zhang R. 2012. Ginsenosides as Anticancer Agents: In vitro and in vivo Activities, Structure-Activity Relationships, and Molecular Mechanisms of Action. Front Pharmacol 3:25. 10.3389/fphar.2012.00025

Nemeth J, Stein I, Haag D, Riehl A, Longerich T, Horwitz E, Breuhahn K, Gebhardt C, Schirmacher P, Hahn M, Ben-Neriah Y, Pikarsky E, Angel P, and Hess J. 2009. S100A8 and $\mathrm{S100A9}$ are novel nuclear factor kappa $B$ target genes during malignant progression of murine and human liver carcinogenesis. Hepatology 50:1251-1262. 10.1002/hep.23099

Plati J, Bucur O, and Khosravi-Far R. 2008. Dysregulation of apoptotic signaling in cancer: molecular mechanisms and therapeutic opportunities. J Cell Biochem 104:1124-1149. $10.1002 / j c b .21707$

Rhyasen GW, Bolanos L, Fang J, Jerez A, Wunderlich M, Rigolino C, Mathews L, Ferrer M, Southall N, Guha R, Keller J, Thomas C, Beverly LJ, Cortelezzi A, Oliva EN, Cuzzola M, Maciejewski JP, Mulloy JC, and Starczynowski DT. 2013. Targeting IRAK1 as a therapeutic approach for myelodysplastic syndrome. Cancer Cell 24:90-104. 10.1016/j.ccr.2013.05.006

Saygin C, Matei D, Majeti R, Reizes O, and Lathia JD. 2019. Targeting Cancer Stemness in the Clinic: From Hype to Hope. Cell Stem Cell 24:25-40. 10.1016/j.stem.2018.11.017

Schettini F, Giuliano M, De Placido S, and Arpino G. 2016. Nab-paclitaxel for the treatment of triple-negative breast cancer: Rationale, clinical data and future perspectives. Cancer Treat Rev 50:129-141. 10.1016/j.ctrv.2016.09.004

Sharifi S, Barar J, Hejazi MS, and Samadi N. 2014. Roles of the Bcl-2/Bax ratio, caspase-8 and 9 in resistance of breast cancer cells to paclitaxel. Asian Pac J Cancer Prev 15:86178622. 10.7314/apjcp.2014.15.20.8617

Shaukat A, Guo YF, Jiang K, Zhao G, Wu H, Zhang T, Yang Y, Guo S, Yang C, Zahoor A, Akhtar M, Umar T, Shaukat I, Rajput SA, Hassan M, and Deng G. 2019. Ginsenoside $\mathrm{Rb} 1$ ameliorates Staphylococcus aureus-induced Acute Lung Injury through attenuating NF-kappaB and MAPK activation. Microb Pathog 132:302-312. 10.1016/j.micpath.2019.05.003

Peer) reviewing PDF | (2020:01:45012:2:0:NEW 3 May 2020) 
418

419

420

421

422

423

424

425

426

427

428

429

430

431

432

433

434

435

436

437

438

439

440

441

442

443

444

445

446

447

448

449

450

451

452

453

454

455

Sun HR, Wang S, Yan SC, Zhang Y, Nelson PJ, Jia HL, Qin LX, and Dong QZ. 2019. Therapeutic Strategies Targeting Cancer Stem Cells and Their Microenvironment. Front Oncol 9:1104. 10.3389/fonc.2019.01104

Taniguchi K, and Karin M. 2018. NF-kappaB, inflammation, immunity and cancer: coming of age. Nat Rev Immunol 18:309-324. 10.1038/nri.2017.142

Teng KY, Han J, Zhang X, Hsu SH, He S, Wani NA, Barajas JM, Snyder LA, Frankel WL, Caligiuri MA, Jacob ST, Yu J, and Ghoshal K. 2017. Blocking the CCL2-CCR2 Axis Using CCL2-Neutralizing Antibody Is an Effective Therapy for Hepatocellular Cancer in a Mouse Model. Mol Cancer Ther 16:312-322. 10.1158/1535-7163.Mct-16-0124

Wang J, Tian L, Khan MN, Zhang L, Chen Q, Zhao Y, Yan Q, Fu L, and Liu J. 2018. Ginsenoside Rg3 sensitizes hypoxic lung cancer cells to cisplatin via blocking of NFkappaB mediated epithelial-mesenchymal transition and stemness. Cancer Lett 415:7385. 10.1016/j.canlet.2017.11.037

Wang XJ, Zhou RJ, Zhang N, and Jing Z. 2019. 20(S)-ginsenoside Rg3 sensitizes human nonsmall cell lung cancer cells to icotinib through inhibition of autophagy. Eur J Pharmacol 850:141-149. 10.1016/j.ejphar.2019.02.023

Warren CFA, Wong-Brown MW, and Bowden NA. 2019. BCL-2 family isoforms in apoptosis and cancer. Cell Death Dis 10:177. 10.1038/s41419-019-1407-6

Wee ZN, Yatim SM, Kohlbauer VK, Feng M, Goh JY, Bao Y, Lee PL, Zhang S, Wang PP, Lim E, Tam WL, Cai Y, Ditzel HJ, Hoon DS, Tan EY, and Yu Q. 2015. IRAK1 is a therapeutic target that drives breast cancer metastasis and resistance to paclitaxel. Nat Commun 6:8746. 10.1038/ncomms9746

Wong AS, Che CM, and Leung KW. 2015. Recent advances in ginseng as cancer therapeutics: a functional and mechanistic overview. Nat Prod Rep 32:256-272. 10.1039/c4np00080c

Yang LQ, Wang B, Gan H, Fu ST, Zhu XX, Wu ZN, Zhan DW, Gu RL, Dou GF, and Meng ZY. 2012. Enhanced oral bioavailability and anti-tumour effect of paclitaxel by 20(s)ginsenoside Rg3 in vivo. Biopharm Drug Dispos 33:425-436. 10.1002/bdd.1806

Yang M, Qin X, Qin G, and Zheng X. 2019. The role of IRAK1 in breast cancer patients treated with neoadjuvant chemotherapy. Onco Targets Ther 12:2171-2180. 10.2147/ott.S185662

Yue PY, Wong DY, Wu PK, Leung PY, Mak NK, Yeung HW, Liu L, Cai Z, Jiang ZH, Fan TP, and Wong RN. 2006. The angiosuppressive effects of 20(R)- ginsenoside Rg3. Biochem Pharmacol 72:437-445. 10.1016/j.bcp.2006.04.034

Zhang S, Zhang H, Ghia EM, Huang J, Wu L, Zhang J, Lam S, Lei Y, He J, Cui B, Widhopf GF, 2nd, Yu J, Schwab R, Messer K, Jiang W, Parker BA, Carson DA, and Kipps TJ. 2019. Inhibition of chemotherapy resistant breast cancer stem cells by a ROR1 specific antibody. Proc Natl Acad Sci U S A 116:1370-1377. 10.1073/pnas.1816262116

Peer) reviewing PDF | (2020:01:45012:2:0:NEW 3 May 2020) 
Figure 1

GPT combined with paclitaxel (PTX) inhibit MB231-PR cell viability and induce cell apoptosis

(A) Single treatment of GPT on MB231-PR cell viability. Cells were treated with different concentration of GPT for 4 days. (B) Combination treatment of GPT and PTX on MB231-PR cell viability. Cells were treated with DMOS, 75 nM PTX, $10 \mu$ M GPT, 75 nM PTX $+2.5 \mu$ M GPT, 75 nM PTX + $5 \mu \mathrm{M}$ GPT, 75 nM PTX + $10 \mu \mathrm{M}$ GPT, respectively. (C) Combination treatment of GPT and PTX on MB231-PT cell viability. Cells were treated with DMSO, $1 \mathrm{nM}$ PTX, $10 \mu \mathrm{M}$ GPT, and different combination, respectively. (D) Representative images of colony formation assay. MB321-PR cells were treated for 12 days with DMSO, 75 nM PTX, $10 \mu M$ GPT and combination, respectively. (E) Flow cytometry detection of cell cycle after treatment for $48 \mathrm{~h}$ and 72 h. $* * \mathrm{P}<0.01, * * * * \mathrm{P}<0.0001$. P-values were calculated with $\mathrm{t}$ test.
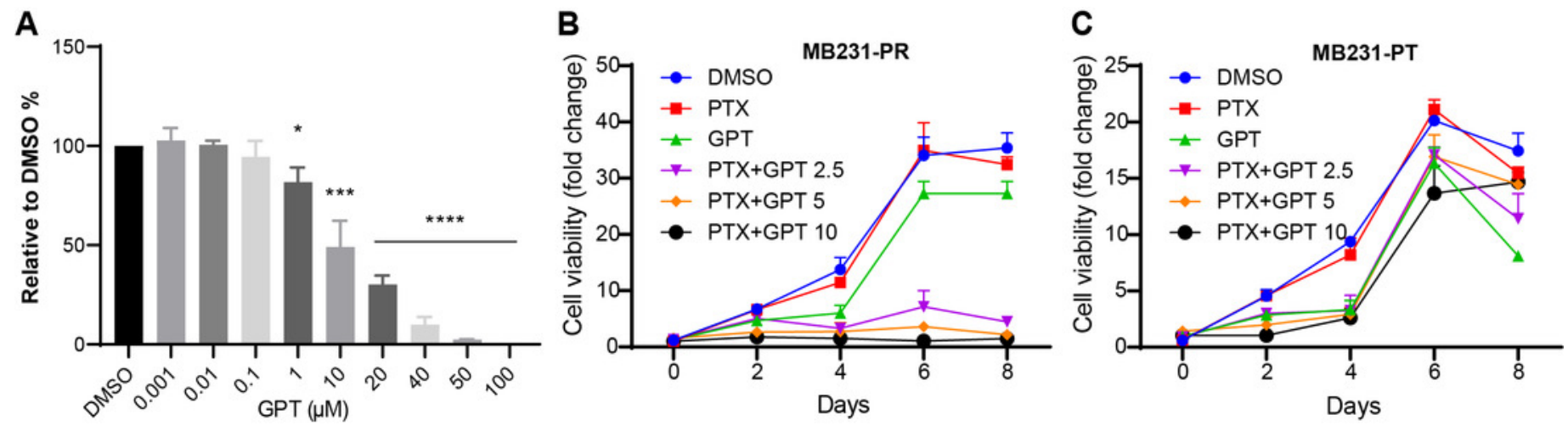

D
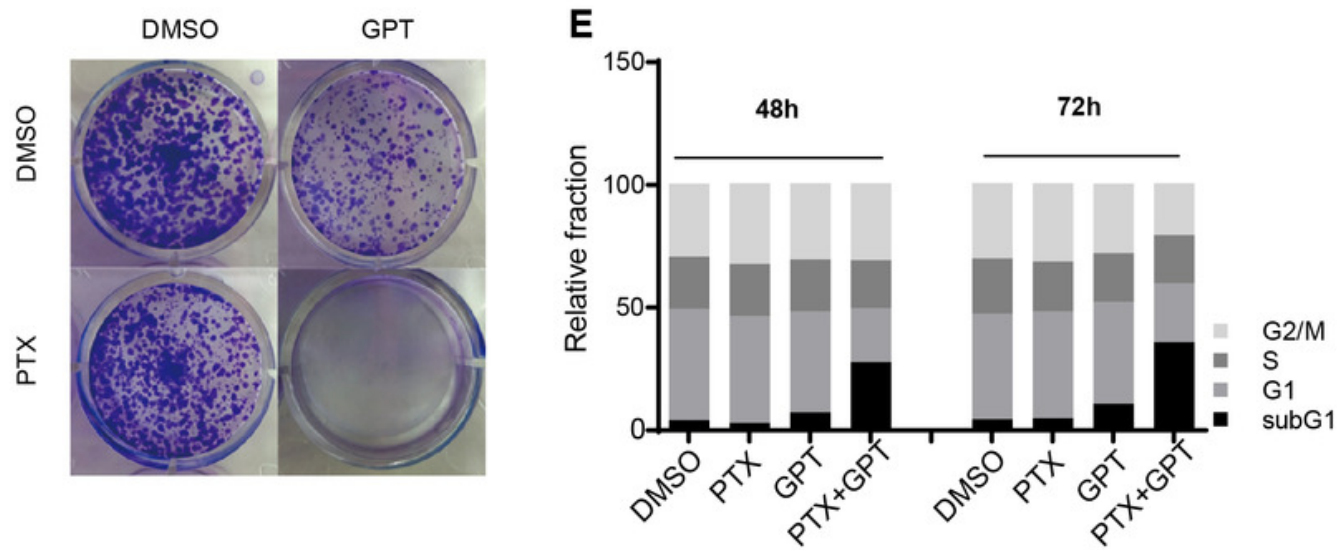


\section{Figure 2}

The combination treatment activates apoptosis pathway and inhibits IRAK1/NF-KB, ERK pathways in MB231-PR cells

(A) Western blot analysis of proteins expression after cells treated with DMSO, 75 nM PTX, $10 \mu \mathrm{M} \mathrm{GPT}$, and different combination for $24 \mathrm{~h}$. (B) Western blot analysis of proteins expression after cells treated with DMSO, $75 \mathrm{nM}$ PTX, $10 \mu \mathrm{M}$ GPT, and combination for $24 \mathrm{~h}$ and $48 \mathrm{~h}$, respectively. (C-H) qPCR analysis of IRAK1/NF-KB downstream inflammatory cytokines and S100A7/9 gene expression after cells treated for $24 \mathrm{~h}$ and $48 \mathrm{~h}$, respectively.*P $<0.05, * * \mathrm{P}<0.01, * * * \mathrm{P}<0.001, * * * * \mathrm{P}<0.0001$. P-values were calculated with $\mathrm{t}$ test. 
A

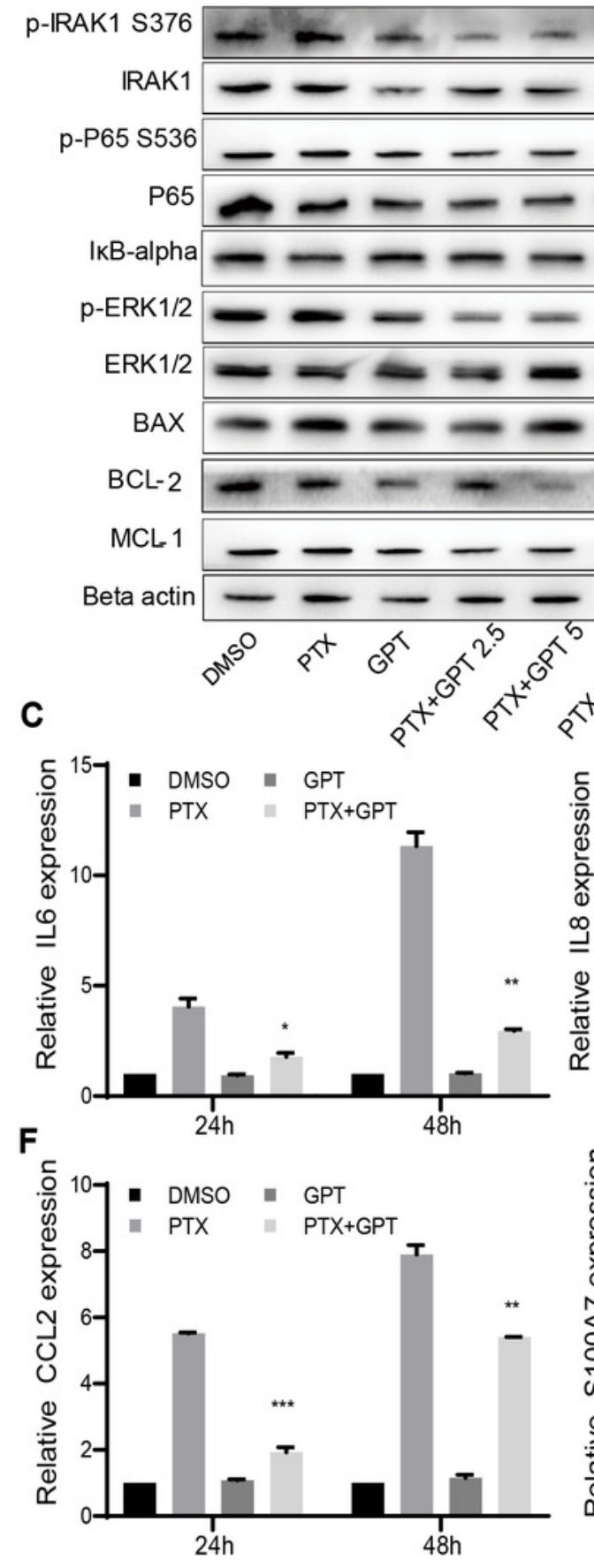

B

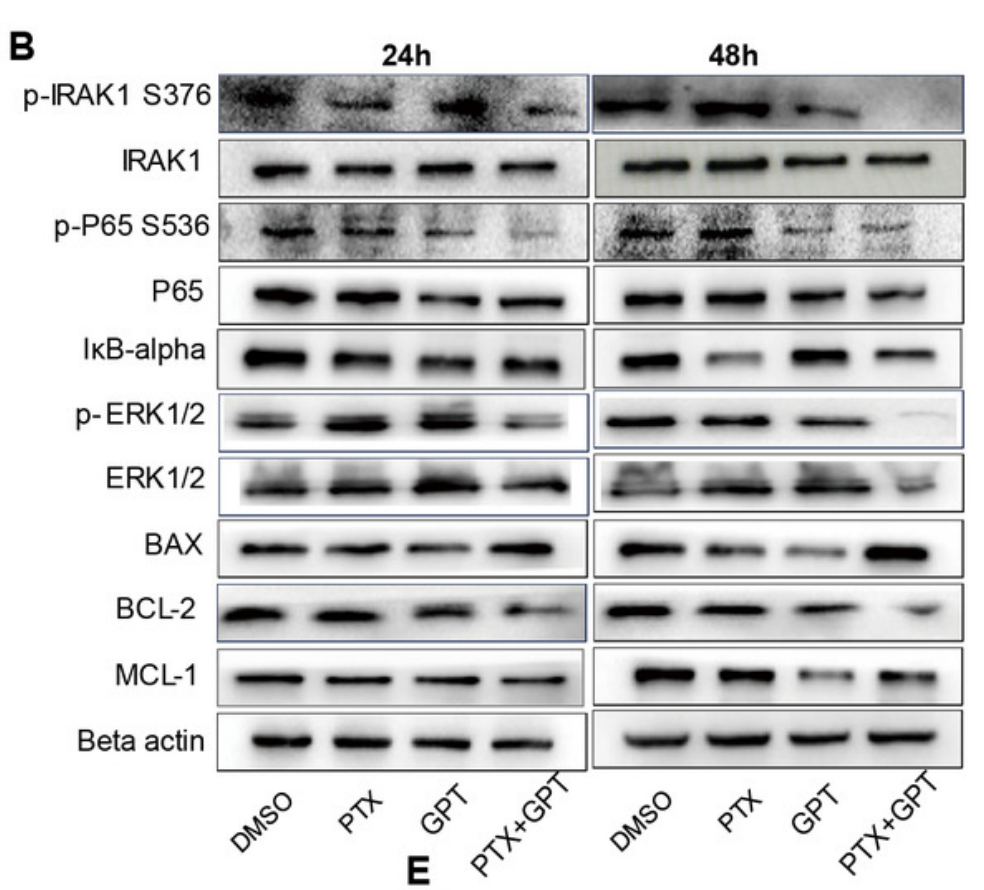

$t^{x} D$

- DMSO = GPT
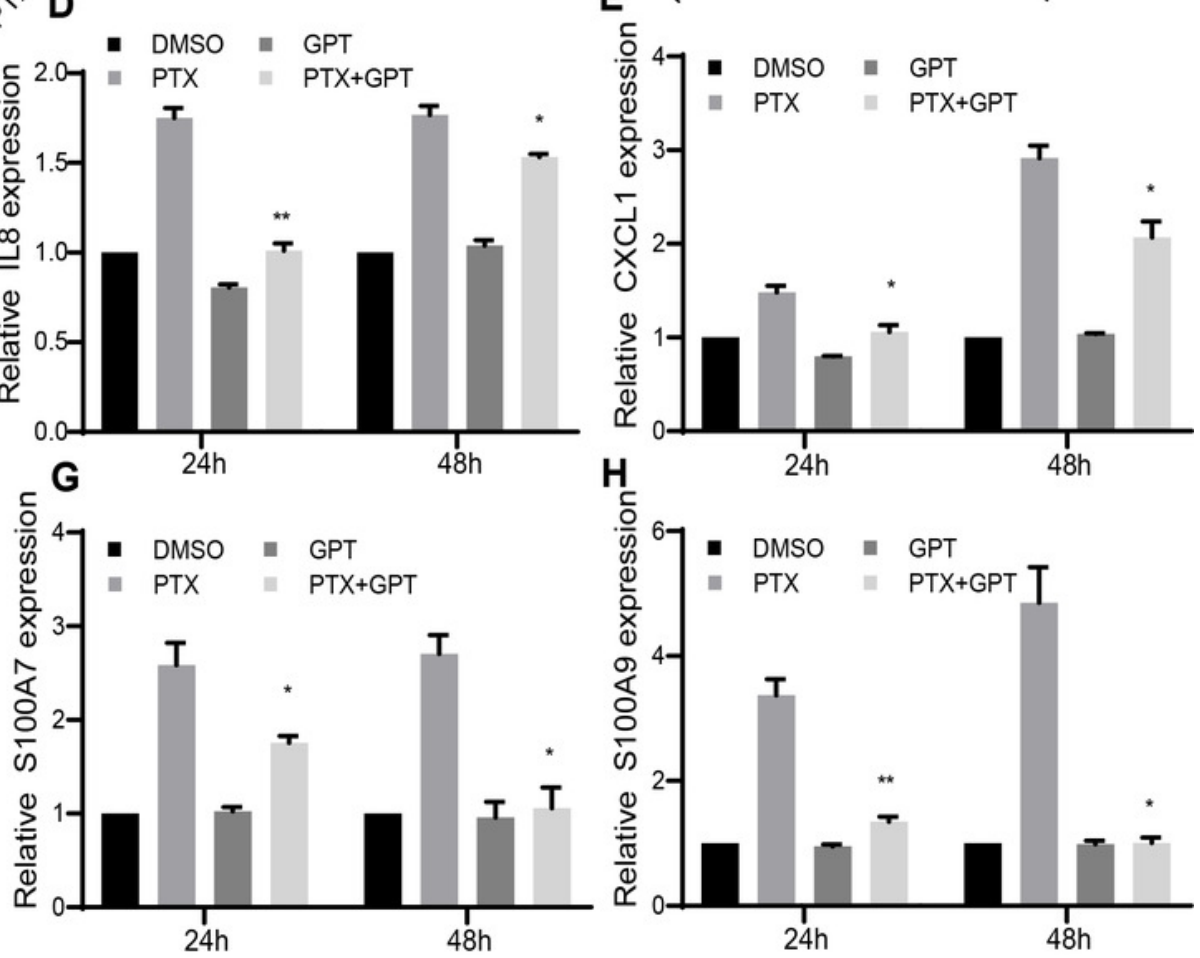


\section{Figure 3}

The combination treatment inhibits inflammatory cytokines expression, tumor sphere growth and cell invasion ability

( A-E) qPCR analysis of CSC-related genes expression after cells treated with DMSO, $75 \mathrm{nM}$ PTX, $10 \mu \mathrm{M}$ GPT, and combination for $24 \mathrm{~h}$ and $48 \mathrm{~h}$, respectively. (F) Transwell invasion assay of MB231-PR cells after drug treatment. Cells were seeded into Corning transwell polycarbonate membrane inserts coated with Matrigel $(300 \mu \mathrm{g} / \mathrm{mL}$ ) and cultured for $24 \mathrm{~h}$. (GJ) Representative images of tumor sphere assays. Cells were seeded into Corning 96-well spheroid microplates and cultured with MammoCul medium. Tumor sphere was observed after treated for 12 days. $* \mathrm{P}<0.05, * * \mathrm{P}<0.01, * * * \mathrm{P}<0.001$. P-values were calculated with $\mathrm{t}$ test. 


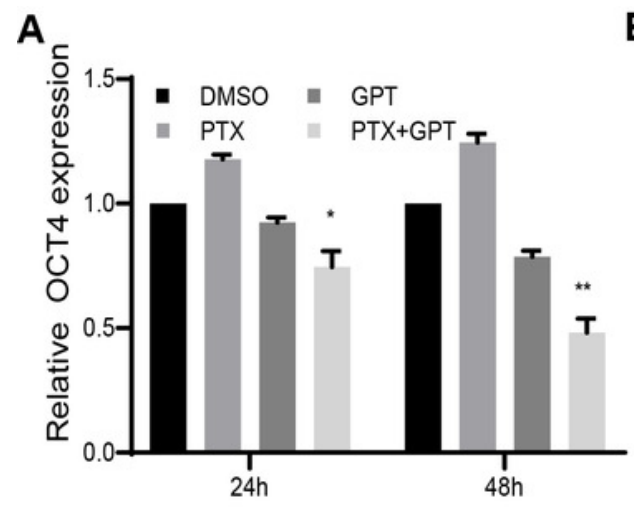

B
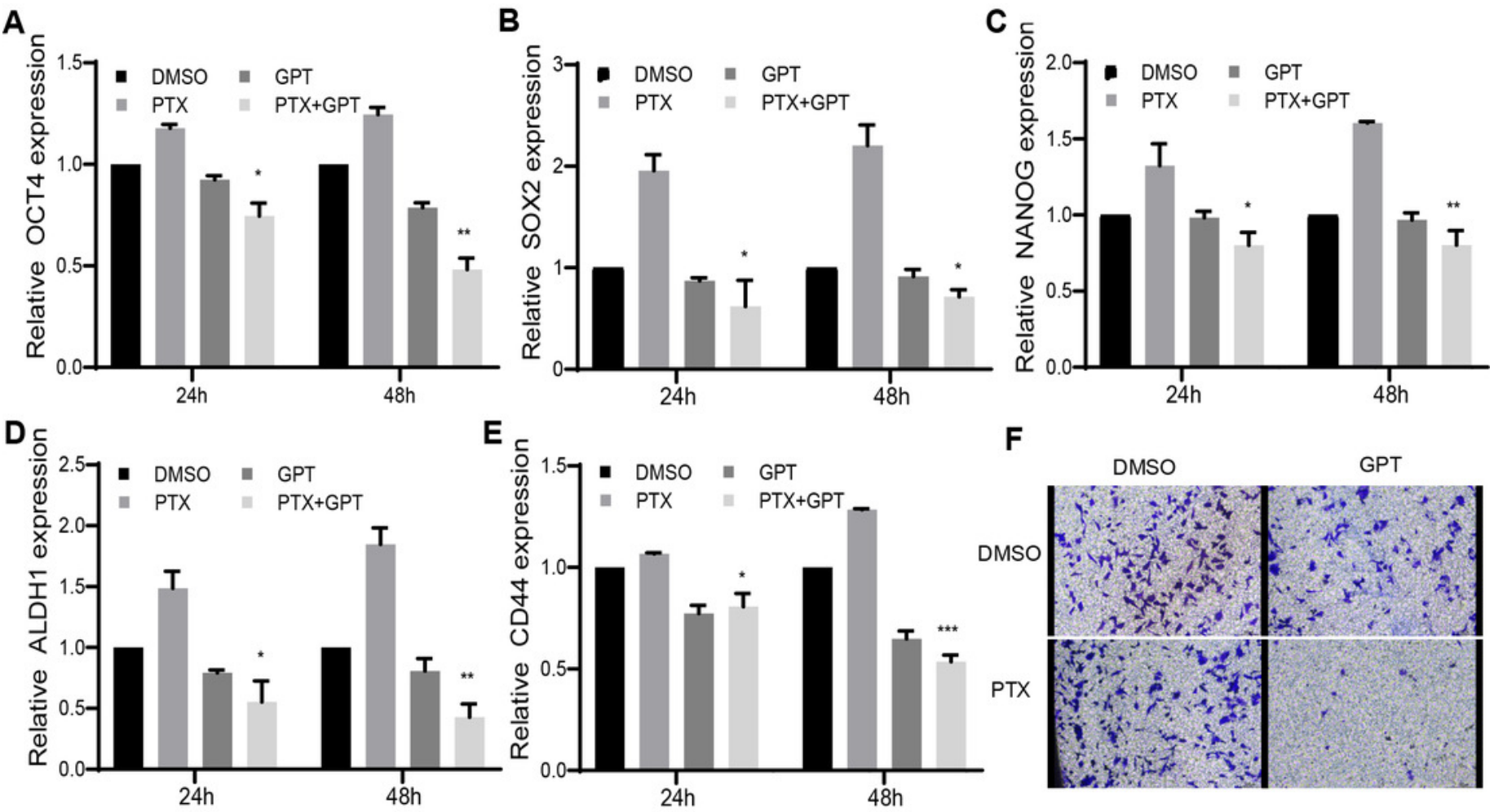

F
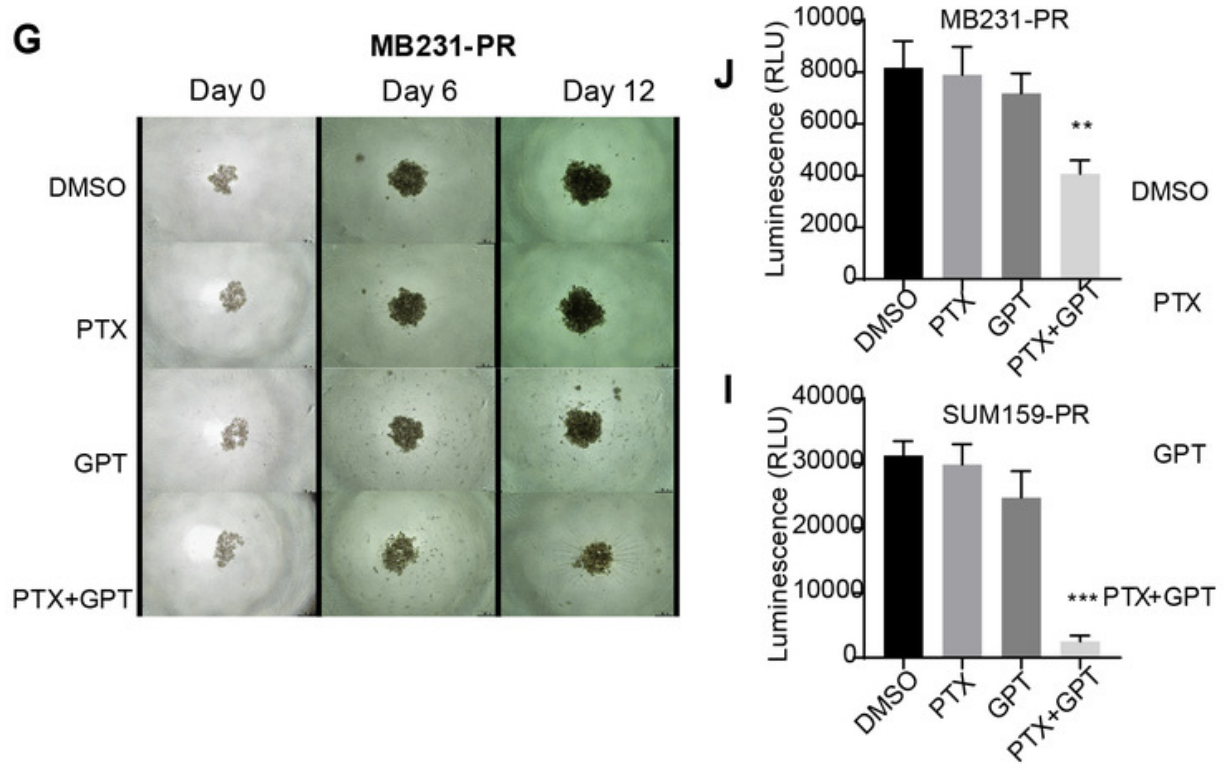

H

SUM159-PR

Day 0

Day 6

Day 12

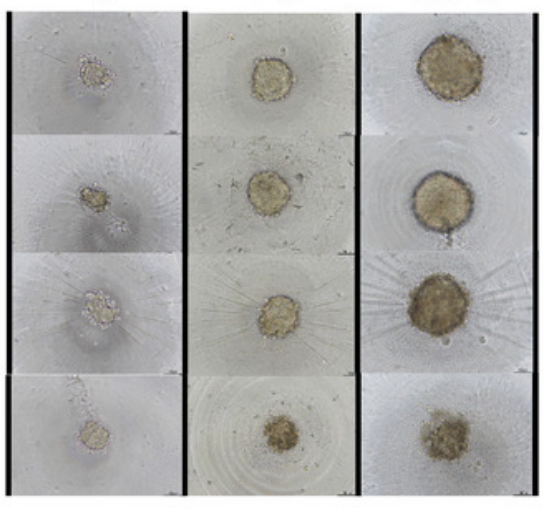


Table $\mathbf{1}$ (on next page)

qPCR primers 
Table 1 qPCR primers

\begin{tabular}{ccc}
\hline Gene & forward (5’'3’) & reverse (5'-3') \\
\hline IL6 & AGTTCCTGCAGAAAAAGGCAAAG & AAAGCTGCGCAGAATGAGAT \\
IL8 & ACCGGAAGGAACCATCTCAC & GGCAAAACTGCACCTTCACAC \\
CXCL1 & CCAGCTCTTCCGCTCCTC & CACGGACGCTCCTGCTG \\
CCL2 & CCCAAAGAAGCTGTGATCTTCA & TCTGGGGAAAGCTAGGGGAA \\
S100A7 & GACAAGATTGAGAAGCCAAGCC & TGTGCCCTTTTTGTCACAGG \\
S100A8 & TGCCGTCTACAGGGATGAC & TCTGCACCCTTTTTCCTGATATAC \\
S100A9 & TCCTCGGCTTTGACAGAGTG & TGGTCTCTATGTTGCGTTCCA \\
OCT4 & CTGGGTTGATCCTCGGACCT & CCATCGGAGTTGCTCTCCA \\
SOX2 & GCCGAGTGGAAACTTTTGTCG & GGCAGCGTGTACTTATCCTTCT \\
NANOG & TTTGTGGGCCTGAAGAAAACT & AGGGCTGTCCTGAATAAGCAG \\
ALDH1 & CTGCTGGCGACAATGGAGT & GTCAGCCCAACCTGCACAG \\
CD44 & TGCCGCTTTGCAGGTGTATT & CCGATGCTCAGAGCTTTCTCC \\
18S & CGAACGTCTGCCCTATCAACTT & ACCCGTGGTCACCATGGTA \\
\hline
\end{tabular}

1 